\title{
Charge Stripes and Antiferromagnetism in Insulating Nickelates and Superconducting Cuprates
}

\author{
J. M. Tranquada \\ Physics Department, Brookhaven National Laboratory, Upton, NY 11973, USA
}

\begin{abstract}
Neutron and X-ray scattering studies have provided strong evidence for coupled spatial modulations of charge and spin densities in layered nickelates and cuprates. The accumulated results for $\mathrm{La}_{2-x} \mathrm{Sr}_{x} \mathrm{NiO}_{4+\delta}$ are consistent with the strongly-modulated topological-stripe concept. Clues from Nd-doped $\mathrm{La}_{2-x} \mathrm{Sr}_{x} \mathrm{CuO}_{4}$ suggest similar behavior for the cuprates. The experimental results are summarized, and features that conflict with an interpretation based on a Fermi-surface instability are emphasized. A rationalization for the differences in transport properties between the cuprates and nickelates is given.
\end{abstract}

\section{Introduction}

Both $\mathrm{La}_{2} \mathrm{NiO}_{4}$ and $\mathrm{La}_{2} \mathrm{CuO}_{4}$ are two-dimensional antiferromagnetic insulators. Their insulating natures are due to the strong Coulomb repulsion $U$ between electrons occupying the same transition-metal $3 d$ orbital. As recently emphasized by Anderson [1], a sufficiently large $U$ can cause the $3 d$ electrons to be localized. Virtual hopping of the electrons between neighboring metal sites lowers their kinetic energy in second order perturbation theory, and when combined with the Pauli exclusion principle, this leads to the antiparallel alignment of neighboring spins.

Various spectroscopic studies have confirmed that $U$ is indeed sufficiently large to cause strong correlations. The fact that the large insulating gaps remain when samples are heated above their respective Néel temperatures further demonstrates that these materials are not Slater antiferromagnets or spindensity-wave systems. Thus, the appropriate starting point for considering the insulating nickelate and cuprate compounds consists of localized electrons with effective interactions via superexchange. 
What we are really interested in, however, is what happens when holes are doped into the $\mathrm{MO}_{2}$ planes $(M=\mathrm{Cu}$ or $\mathrm{Ni})$. In the case of the cuprate, a hole concentration $n_{h} \gtrsim 0.05$ per $\mathrm{Cu}$ yields an unusual metal that becomes superconducting at surprisingly high temperatures. The nickelate, on the other hand, remains insulating even when $n_{h}$ is increased to 0.5 . Such strongly contrasting behavior might lead one to expect that the electronic correlations in these systems evolve in quite different manners; however, neutron scattering studies have shown that the magnetic (and possibly charge) correlations follow very similar trends with doping. In this paper I will review these trends, and consider the relevance of various theoretical approaches for interpreting them.

\section{$2 \quad \mathbf{L a}_{2-x} \mathrm{Sr}_{x} \mathrm{NiO}_{4+\delta}$}

Consider first the nickelates. At low doping it appears that the holes must be localized near the dopants. As a result, the details of the phase diagram in this regime depend on the nature of the dopant, substituted $\mathrm{Sr}$ or excess oxygen. Once the hole concentration is large enough, $n_{h} \gtrsim 0.2$, coupled chargeand spin-density modulations start to form within the $\mathrm{NiO}_{2}$ planes. These modulations align themselves diagonally with respect to the Ni-O bonds, as indicated by the positions of the charge-order and magnetic superlattice peaks observed by electron [2] and neutron diffraction [3-5]. If one makes use of a unit cell with axes rotated by $45^{\circ}$ with respect to the $\mathrm{Ni}-\mathrm{O}$ bonds, then in reciprocal space the magnetic peaks are split about the antiferromagnetic position $(1,0,0)$ along the [100] and [010] directions by an amount $\epsilon$ (in reciprocal lattice units), while the charge-order peaks are split about fundamental Bragg peaks by $2 \epsilon$. (Neutrons do not scatter from charge directly, but instead are sensitive to the atomic displacements induced by the charge-density modulation. The chargeorder peaks have also been observed by X-ray diffraction [6].) The relationship between charge and spin wave vectors indicates that the period of the spin structure in real space is twice that of the charge modulation. Measurements of higher-order magnetic harmonics [7] and the magnitude of magnetic moments

[3] are consistent with a strong localization of the charge into stripes of the type suggested by calculations of Zaanen and Littlewood [8]. In this picture, the regions between stripes are antiferromagnetic domains, with a phase that shifts by $\pi$ on crossing a stripe. Experiments on a range of samples show that $\epsilon \approx n_{h}=x+2 \delta$ at low temperature. This indicates that adding more holes results in more stripes of roughly constant charge density (roughly 1 hole/Ni) which must move closer together.

The relationship between charge-order and magnetic-order wave vectors is just the same as that found in $\mathrm{Cr}$ [9]. In the latter system, the dominant spindensity wave (SDW) order is consistent with Fermi-surface nesting. The weak charge order appears at the same temperature as the SDW, and the CDW 


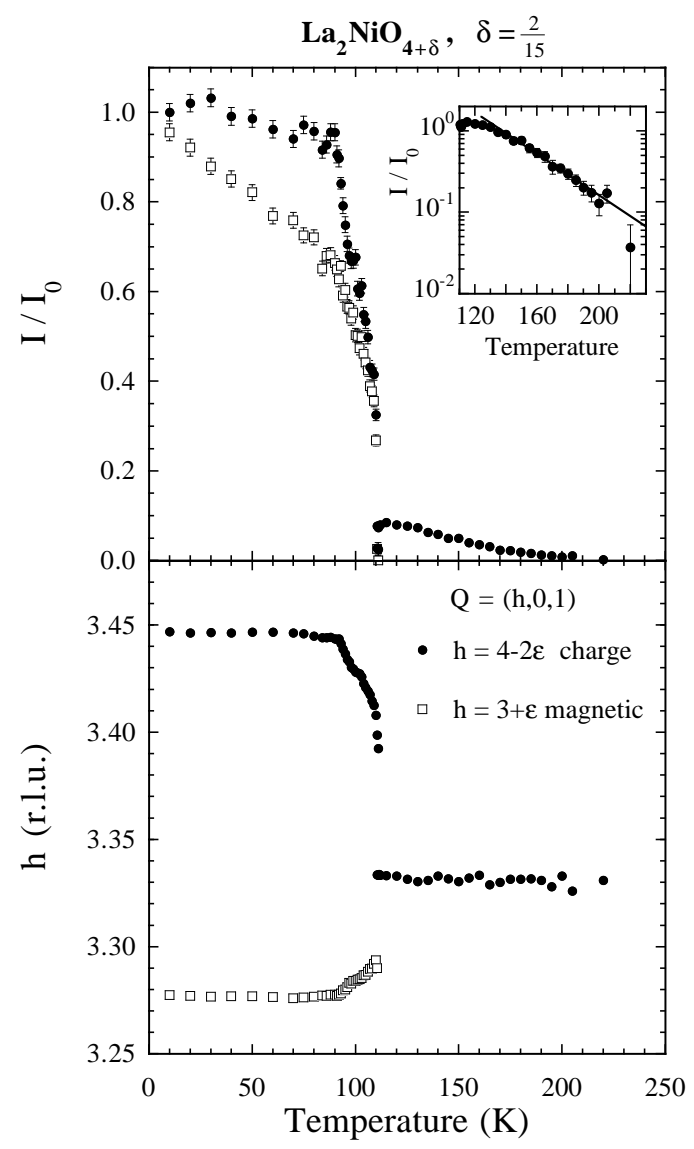

Fig. 1. Temperature dependences of a magnetic peak at $(3+\epsilon, 0,1)$ (open squares) and a charge order peak at $(4-2 \epsilon, 0,1)$ (filled squares) measured by neutron diffraction on a single crystal of $\mathrm{La}_{2} \mathrm{NiO}_{4.133}$. Upper panel shows integrated intensities normalized at $10 \mathrm{~K}$; lower gives the peak position in $h$. From Wochner et al. [7].

order parameter varies as the square of that for the SDW. In the nickelates, the situation is rather different. The charge order always appears at a higher temperature than the magnetic order, and both ordering temperatures tend to increase linearly with $n_{h}$. Such behavior is inconsistent with order driven purely by the spin density [10]. Furthermore, optical reflectivity measurements on a Sr-doped sample with $x=\frac{1}{3}$ indicate that the $\mathrm{NiO}_{2}$ planes do not become metallic even at temperatures well above the ordering temperature [11].

Other relevant results have been obtained in a recent neutron diffraction study of $\mathrm{La}_{2} \mathrm{NiO}_{4+\delta}$ with $\delta=\frac{2}{15}$ [7]. As shown in the lower panel of Fig. 1, for this sample $\epsilon$ is temperature dependent; more precise measurements of a thirdharmonic peak position reveal lock-in plateaus at certain rational fractions. In the case of Fermi-surface-driven order, one would expect the wave vector to lock in at a temperature-independent value, $2 k_{F}$. In contrast, the observed behavior suggests that competing interactions are involved, one of which is likely to be the long-range part of the Coulomb interaction. 


\section{$3 \quad \mathrm{La}_{2-x} \mathrm{Sr}_{x} \mathrm{CuO}_{4}$}

Evidence for incommensurate magnetic fluctuations in $\mathrm{La}_{2-x} \mathrm{Sr}_{x} \mathrm{CuO}_{4}$ was first provided by the inelastic neutron scattering measurements of Cheong et al. [12]. To describe this it is convenient to express results in terms of a unit cell with axes parallel to the $\mathrm{Cu}-\mathrm{O}$ bonds, so that the antiferromagnetic wave vector is $\left(\frac{1}{2}, \frac{1}{2}, 0\right)$. The magnetic scattering is peaked at positions split about this wave vector by an amount $\epsilon$ along [100] and [010] directions. Yamada et al. [13] have shown that $\epsilon \approx x$ for $0.05 \lesssim x \lesssim \frac{1}{8}$, and $\epsilon \approx \frac{1}{8}$ for $x \gtrsim \frac{1}{8}$.

The fact that $\mathrm{La}_{2-x} \mathrm{Sr}_{x} \mathrm{CuO}_{4}$ is metallic has caused some researchers to look to $\mathrm{Cr}$ as a model for understanding the incommensurate magnetic scattering [14]. While it is true that $\mathrm{Cr}$ alloys exhibit similar magnetic scattering that can be purely inelastic or have an $E=0$ component [15], these alloys always tend to be good metals, and it is not possible to achieve a correlated insulator state by doping. In contrast, many of the experimentally observed features in the cuprates, such as the lack of a shift in the chemical potential in $\mathrm{La}_{2-x} \mathrm{Sr}_{x} \mathrm{CuO}_{4}$ for $x \lesssim 0.15$ [16], bear strong similarities to doped semiconductors; hence, proximity to an insulating phase appears to be an important feature of the phase diagram. Recently it has been argued that a generic feature of $2 \mathrm{D}$ doped insulators is the generation of topological defects in the form of antiphase domain walls $[17,18]$. In the case of doped $\mathrm{La}_{2} \mathrm{NiO}_{4}$, we have seen that these topological stripes have scattering signatures similar to those of $\mathrm{Cr}$ alloys even though the underlying physics appears to be different.

To gain further clues on the nature of the magnetic correlations in the cuprates, studies have been performed on $\mathrm{La}_{1.6-x} \mathrm{Nd}_{0.4} \mathrm{Sr}_{x} \mathrm{CuO}_{4}$. The substituted $\mathrm{Nd}$ has the same valence but a smaller ionic radius than the La, and the latter feature induces a subtle change in the low-temperature tilt pattern of the $\mathrm{CuO}_{6}$ octahedra $[19,20]$. Associated with this structural modification is a strong suppression of $T_{c}$ for $x \approx \frac{1}{8}$. Neutron diffraction studies [21,22] on a single crystal with $x=0.12$ have shown the appearance of elastic magnetic scattering with the same incommensurate splitting $\epsilon$ as that found in crystals with the same $x$ and no Nd [13]. Furthermore, weak peaks consistent with charge order have been found with neutrons $[21,22]$ and confirmed with high-energy Xray scattering [23]. The charge order appears at a higher temperature than the magnetic order, as in the nickelates. Further work has shown that the static magnetic order coexists with superconductivity in Nd-doped crystals with $x=0.15$ and $0.20[24,25]$.

At first glance, one might guess that the depression of $T_{c}$ in the Nd-doped crystals might be due to a change in the density of states near $E_{F}$ associated with the modification of the crystal structure; however, band calculations based on experimentally determined crystal structures indicate that the phase change 
has relatively little effect on the density of states [26]. If the magnetic and charge order were driven by a Fermi surface instability, it would have to be a CDW-type instability because of the distinct ordering temperatures [10]; however, the wave vector for the charge order $(2 \epsilon=0.24 \mathrm{rlu})$ is not a special spanning vector of the Fermi surface. Also, the photoemission measurements on $\mathrm{La}_{2-x} \mathrm{Sr}_{x} \mathrm{CuO}_{4}$ reported by Fujimori et al. [27] do not provide any evidence for coherent quasiparticles near $E_{F}$; such quasiparticles would be a prerequisite for any Fermi-surface-driven ordering. Finally, optical reflectivity measurements on $\mathrm{Nd}$-doped crystals [28] indicate that the charge-excitation gap one would expect in the case of a CDW would have to be exceedingly small.

If the spin and charge modulations are related to topological stripes, then one would expect the amplitudes of the modulations to be large. Although direct measurement of the amplitude of the charge modulation is difficult, local-probe measurements of the spin amplitude are available [29-31]. In particular, muon spin rotation $(\mu \mathrm{SR})$ studies on samples of $\mathrm{La}_{2-x-y} \mathrm{Nd}_{y} \mathrm{Sr}_{x} \mathrm{CuO}_{4}$, with $x=0.12$, $y=0.4$ [29], and with $x=0.15, y=0.3-0.6$ [30], indicate a maximum ordered moment approximately half that in $\mathrm{La}_{2} \mathrm{CuO}_{4}$, and an ordering temperature of $\sim 30 \mathrm{~K}$. The magnitude of the ordered moments is substantially reduced compared to the antiferromagnetic insulator state, but at the same time, the ordering temperature is diminished by an even greater amount. One might expect quantum spin fluctuations to affect both quantities, but how can we evaluate such effects? Castro Neto and Hone [32] have proposed that the main effect of the stripes is to reduce the exchange coupling between neighboring magnetic domains, which can be modelled in terms of a spatially anisotropic nonlinear sigma model. Using such a model, van Duin and Zaanen [33] have calculated the ordering temperature $T_{N}$ (which requires an assumption about interlayer coupling) and low-temperature staggered magnetization $M_{s}$ as a function of anisotropy, $\alpha$, where $\alpha=1$ corresponds to isotropic interactions and the local superexchange is held constant. Although the magnitude of the effective anisotropy is not known, a useful way to present the results is to plot the variation of $T_{N}(\alpha)$ versus $M_{s}(\alpha)$, as shown in Fig. 2. Normalizing the experimental results on the modulated phase to those for $\mathrm{La}_{2} \mathrm{CuO}_{4}$, we see that they are fairly consistent with this model. Thus, the observed spin-density amplitude is compatible with the strong modulation expected for topological stripes.

The simplest caricature of ordered charge stripes involves a non-sinusoidal modulation of the charge and spin densities. The stripe interpretation has been questioned due to the lack of evidence for strong higher-harmonic superlattice peaks that might be expected. It turns out, however, that such a concern is not relevant. First of all, as demonstrated in the case of a nickelate sample [7], the intensities of higher harmonics corresponding to narrow stripes are actually quite weak and would be difficult to observe in the cuprates. Secondly, 




Fig. 2. Solid line: theoretical variation of the relative Néel temperature vs. low-temperature staggered magnetization as an implicit function of exchange anisotropy associated with stripes. From a model calculation by van Duin and Zaanen [33]. The point labelled $\mathrm{La}_{1.48} \mathrm{Nd}_{0.4} \mathrm{Sr}_{0.12} \mathrm{CuO}_{4}$ is from a $\mu \mathrm{SR}$ experiment by Luke et al. [29].

fluctuations of stripes about their average positions should smear the average distribution, leading to a further reduction in harmonic intensities. Thus, the absence of harmonics is not a valid objection to stripes, but at the same time, models with sinusoidal modulations, such as those discussed by Scalapino [34], are also compatible with experiment.

\section{Dynamic Correlations}

For a stripe-ordered phase, the low-energy spin fluctuations have a simple interpretation: they are just the spin waves that one expects in a magnetically ordered state. Inelastic neutron scattering measurements on $\mathrm{La}_{2} \mathrm{NiO}_{4.133}$ appear consistent with this concept [35]. The effective spin-wave velocity is reduced by about $40 \%$ compared to the undoped phase, where the velocity is proportional to the superexchange $J$ times the lattice parameter $a$. The softening of the spin waves in the stripe-ordered phase is consistent with a reduction in the effective value of $J$ due to relatively weak exchange coupling across domain walls [32]; however, the anisotropy that one would expect to see in the spin-wave velocity for propagation parallel and perpendicular to the stripes has not been clearly observed. A quantitative test requires more detailed theoretical predictions. 
The important feature here is that the observed spin fluctuations are associated with the magnetic moments in the antiferromagnetic domains, but not directly with the spins of the doped holes. When static stripe order disappears, one would expect dynamic correlations to survive. Low-energy spin fluctuations should become overdamped, while modes at high enough energy (sufficiently short wavelength) would be relatively insensitive to the loss of order [36]. For $\mathrm{La}_{2-x} \mathrm{Sr}_{x} \mathrm{CuO}_{4}$ with $x=0.14$, the effective spin-wave velocity associated with high-energy fluctuations appears to be reduced compared to the pure antiferromagnet [37], as occurs in the stripe-ordered nickelate discussed above. In contrast, if the spin fluctuations were related to the response of itinerant electrons near the Fermi surface, the velocity should be comparable to the Fermi velocity $v_{F}$ instead of $J a / \hbar$. Band-structure calculations [38] indicate that $v_{F}$ is 2 to 3 times the spin-wave velocity of $\mathrm{La}_{2} \mathrm{CuO}_{4}$ [39].

In $\mathrm{La}_{2-x} \mathrm{Sr}_{x} \mathrm{CuO}_{4}$ with $x=0.14$, where no static stripe order is observed at finite temperature, the dynamics of the magnetic fluctuations might exhibit quantum critical behavior. In fact, evidence for such behavior is reported by Aeppli et al. [40]. A distinct quantum critical point, associated with an incommensurate CDW instability, has been discussed by Di Castro and coworkers $[41]$.

Of course, any correlations that are relevant to the superconductivity must be common to all of the superconducting cuprates. For this reason, the recent observations of incommensurate magnetic scattering in $\mathrm{YBa}_{2} \mathrm{Cu}_{3} \mathrm{O}_{6.6}$, discussed by Mook [42], are quite important. Connections suggested by the response to Zn doping have been listed elsewhere [43]. As an aside to the question of universality, it is interesting to note that stripe modulations have also been observed in electron diffraction studies of doped manganites [44].

\section{Differences Between Cuprates and Nickelates}

I have argued that there are strong similarities between the spatial correlations of charge and spin in the cuprates and nickelates; however, as mentioned in the introduction, the nickelates remain insulating at dopant concentrations where the cuprates become metallic and superconducting. If the hole concentration in the nickelate stripes were precisely 1 hole/ Ni site, then this would be equivalent to a half-filled 1D system. Such a system should be a Mott insulator [1]. However, this explanation is incomplete in the case of $\mathrm{La}_{2} \mathrm{NiO}_{4.133}$ where it has been demonstrated that the charge density within the stripes must vary substantially with temperature [7]. The deviation from $\frac{1}{2}$-filling in these nickelate stripes makes them similar to the cuprate stripes, which, when not pinned to the lattice, apparently participate in metallic transport at finite temperatures. Thus it may not be possible to understand the differences be- 

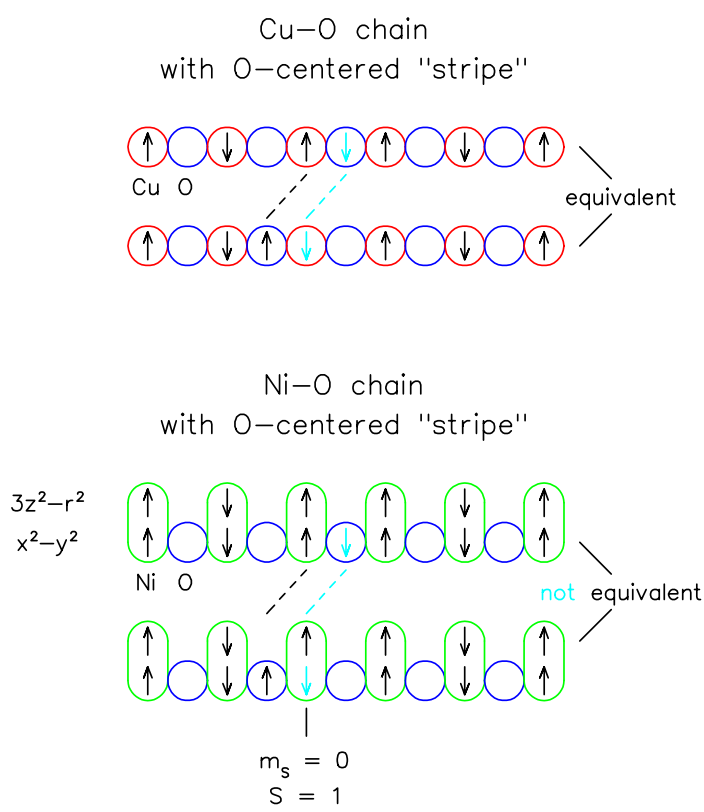

Fig. 3. 1D models of a topological charge stripe for $\mathrm{Cu}-\mathrm{O}$ (top) and $\mathrm{Ni}-\mathrm{O}$ (bottom), as discussed in the text.

tween the nickelates and cuprates simply in terms of the conductivity along a stripe. An alternative approach is to consider the motion of charge in the direction transverse to the stripes.

Let us consider a 1D model for a charge stripe between antiferromagnetic domains. The cuprate case is shown at the top of Fig. 3, with the hole centered on an oxygen atom. The coupling between the $\mathrm{O}$ hole spin and the neighboring $\mathrm{Cu}$ spins is responsible for the antiphase relationship between the magnetic domains. (A Cu-centered domain wall could be described as a superposition of two such O-centered stripes.) If the hole on $\mathrm{O}$ and a neighboring $\mathrm{Cu}$ hole both shift one position to the left, the resulting configuration is equivalent to the original one. Thus, in this simple picture, the $1 \mathrm{D}$ stripe would be expected to be itinerant.

The situation changes in the nickelate case (bottom of Fig. 3) because of the second $3 d$ hole on each $\mathrm{Ni}$. The two $3 d$ spins per $\mathrm{Ni}$ couple in a high-spin triplet state. Now when the $\mathrm{O}$ hole plus a $3 d_{x^{2}-y^{2}}$ hole shift to the left, we end up with a $\mathrm{Ni}$ site in a $m_{s}=0, S=1$ state [45]. This shifted configuration is not equivalent to the original. The shifted state is higher in energy, and hence the stripe will tend to be confined in its original position. This effect should not depend on long-range order, and hence may be relevant even when charge stripes become thermally disordered. 


\section{Acknowledgments}

I have benefited from interactions with many experimental collaborators, including J. D. Axe, D. J. Buttrey, G. Shirane, N. Ichikawa, S. Uchida, and P. Wochner. I am also grateful for frequent discussions with V. J. Emery and S. A. Kivelson. This work is supported by Contract No. DE-AC02-76CH00016, Division of Materials Sciences, U.S. Department of Energy.

\section{References}

[1] P. W. Anderson, Adv. Phys. 46, 3 (1997).

[2] C. H. Chen, S.-W. Cheong, and A. S. Cooper, Phys. Rev. Lett. 71, 2461 (1993).

[3] J. M. Tranquada, J. E. Lorenzo, D. J. Buttrey, and V. Sachan, Phys. Rev. B $\mathbf{5 2}, 3581$ (1995).

[4] J. M. Tranquada, D. J. Buttrey, and V. Sachan, Phys. Rev. B 54, 12318 (1996).

[5] S.-H. Lee and S.-W. Cheong, Phys. Rev. Lett. 79, 2514 (1997).

[6] A. Vigliante et al., Phys. Rev. B 56, 8248 (1997).

[7] P. Wochner, J. M. Tranquada, D. J. Buttrey, and V. Sachan, Phys. Rev. B (in press); cond-mat/9706261.

[8] J. Zaanen and P. B. Littlewood, Phys. Rev. B 50, 7222 (1994).

[9] R. Pynn, W. Press, S. M. Shapiro, and S. A. Werner, Phys. Rev. B 13, 295 (1976).

[10] O. Zachar, V. J. Emery, and S. A. Kivelson, Phys. Rev. B (in press).

[11] T. Katsufuji et al., Phys. Rev. B 54, R14230 (1996).

[12] S.-W. Cheong et al., Phys. Rev. Lett. 67, 1791 (1991).

[13] K. Yamada et al., Physica C 282-287, 85 (1997).

[14] T. E. Mason, G. Aeppli, and H. A. Mook, Phys. Rev. Lett. 68, 1414 (1992).

[15] E. Fawcett et al., Rev. Mod. Phys. 66, 25 (1994).

[16] A. Ino et al., Phys. Rev. Lett. 79, 2101 (1997).

[17] S. A. Kivelson and V. J. Emery, Synth. Met. 80, 151 (1996).

[18] J. Zaanen, J. Phys. Chem. Solids, this conference; cond-mat/9711009.

[19] M. K. Crawford et al., Phys. Rev. B 44, 7749 (1991). 
[20] B. Büchner, M. Breuer, A. Freimuth, and A. P. Kampf, Phys. Rev. Lett. 73, 1841 (1994).

[21] J. M. Tranquada et al., Nature 375, 561 (1995).

[22] J. M. Tranquada et al., Phys. Rev. B 54, 7489 (1996).

[23] M. von Zimmermann et al., (preprint).

[24] J. M. Tranquada et al., Phys. Rev. Lett. 78, 338 (1997).

[25] J. E. Ostenson et al., Phys. Rev. B 56, 2820 (1997).

[26] M. R. Norman, G. J. McMullan, D. L. Novikov, and A. J. Freeman, Phys. Rev. B 48, 9935 (1993).

[27] A. Fujimori et al., J. Phys. Chem. Solids, this conference.

[28] S. Tajima et al., J. Phys. Chem. Solids, this conference.

[29] G. M. Luke et al., Hyp. Int. 105, 113 (1997).

[30] W. Wagener et al., Phys. Rev. B 55, R14 761 (1997).

[31] M. Roepke et al., J. Phys. Chem. Solids, this conference.

[32] A. H. Castro Neto and D. Hone, Phys. Rev. Lett. 76, 2165 (1996).

[33] C. N. A. van Duin and J. Zaanen, cond-mat/9707195.

[34] D. J. Scalapino, J. Phys. Chem. Solids, this conference.

[35] J. M. Tranquada, P. Wochner, and D. J. Buttrey, Phys. Rev. Lett. 79, 2133 (1997).

[36] J. M. Tranquada, Physica C 282-287, 166 (1997).

[37] S. M. Hayden et al., Phys. Rev. Lett. 76, 1344 (1996).

[38] P. B. Allen, W. E. Pickett, and H. Krakauer, Phys. Rev. B 36, 3926 (1987).

[39] G. Aeppli et al., Phys. Rev. Lett. 62, 2052 (1989).

[40] G. Aeppli et al., Science (in press).

[41] C. Di Castro, J. Phys. Chem. Solids, this conference.

[42] H. A. Mook, J. Phys. Chem. Solids, this conference.

[43] J. M. Tranquada, Physica B (in press); cond-mat/9709325.

[44] S.-W. Cheong, J. Phys. Chem. Solids, this conference.

[45] J. Zaanen, (private communication). 